\title{
Vosotros a la taberna, ustedes al boliche (Catulo, Carmen 37) ${ }^{1}$
}

Gabriela Andrea Marrón ${ }^{2}$

CONICET - Universidad Nacional del Sur

\section{Resumen}

En este trabajo proponemos revisar los presupuestos de neutralidad subyacentes tras la adopción del pronombre de segunda persona plural «vosotros» en dos traducciones latinoamericanas del carmen 37 de Catulo: la de Rubén Bonifaz Nuño (1969) y la de Lía Galán (2008). A partir de la convergencia entre el uso pronominal señalado en ambas versiones y el presente en las principales ediciones castellanas del corpus catuliano publicadas en España entre 1950 y 2008, intentaremos mostrar el curioso contraste generado en los otros dos textos, por la inclusión de ciertos términos propios de la variedad lingüística de sus traductores: tales como la connotación sexual del verbo «coger», en el de Galán, y la presencia del diminutivo «chiquito», en el de Bonifaz Nuño. A su vez, confrontaremos ambas traducciones con el resultado obtenido en otras dos versiones del mismo poema, publicadas recientemente en Chile (Sanhueza, 2013) y Argentina (Marrón, 2012), en las que la inclusión de léxico característico de las variedades de cada país coexiste con la elección morfológica del pronombre de segunda persona plural «ustedes» en lugar de «Vosotros». Por último, intentaremos establecer un vínculo entre el fenómeno descripto en estas últimas dos traducciones y el observable en algunas de las publicadas en la primera década del siglo XXI en España -como la de Rosario González Galicia (2002) y la de José Antonio González Iglesias (2006)- en las que el uso del léxico propio de la variedad peninsular impide toda posible inscripción de

\footnotetext{
${ }^{1}$ Este trabajo, desarrollado en el marco de mis actividades como Investigadora Asistente del CONICET, ha sido posible gracias a los subsidios para investigación otorgados por la ANPCyT (PICT $2013 \mathrm{~N}^{\circ} 405$ ), por el CONICET (PIP 2014-2016 No 00089), y por la SGCyT de la Universidad Nacional del Sur (PGI 24/I 227). Agradezco al Dr. Francisco García Jurado, de la Universidad Complutense de Madrid, sin cuya invaluable auxilio me hubiera resultado imposible consultar muchas de las ediciones españolas de la obra de Catulo citadas en este trabajo.

${ }^{2}$ Investigadora Asistente del CONICET. Docente Auxiliar en las cátedras de Cultura Clásica y de Lengua y Cultura Latina I del Departamento de Humanidades de la Universidad Nacional del Sur.
} 
los textos resultantes en el constructo lingüístico -pretenciosamente- panhispánico denominado «español neutro».

\title{
Palabras clave
}

Catulo - léxico obsceno - usos pronominales - traducción

\begin{abstract}
The purpose of this article is to examine the neutrality assumptions lying behind the adoption of the second personal plural pronoun «vosotros» in two Latin-American translations of Catullus' Carmen 37: one published by Rubén Bonifaz Nuño (1969) and the other by Lía Galán (2008). Although the signaled pronominal use occurs not only in those two texts, but also in the principal Spanish editions of Catullus' corpus published in Spain between 1950 and 2008, we shall attempt to point at a particular contrast present in those two versions, where the pronoun «vosotros» coexist with lexical elements of the translators' linguistic varieties: as the sexual connotation of the verb «coger», in Galan's, or the use of the diminutive «chiquito» in Bonifaz Nuño's. In addition, we intend to confront both translations with other two Latin-American versions of the same poem recently published in Chile (Sanhueza, 2013) and Argentina (Marrón, 2012), in which the inclusion of lexical items belonging to the translators' linguistic varieties concur with the morphologic choice of the second personal plural pronoun «ustedes» in detriment of «vosotros». Finally, we shall attempt to link together the interesting phenomenon described in these Chilean and Argentinian versions with the similar trend noticeable in some of the translations published in Spain during the first ten years of the 21st century -as those made by Rosario González Galicia (2002) and by José Antonio González Iglesias (2006)-, where the presence of lexical elements belonging to the Peninsular Spanish variety prevents any possible inscription of the resultant texts in the linguistic -pretentiously- Pan-Hispanic construction designated as «neutral Spanish».
\end{abstract}

\section{Key Words}

Catullus - obscene lexicon - pronominal usage - translation 
Si traducir un poema de Catulo al español plantea el desafío de trasladarlo a una lengua diferente a la latina, pero a la vez desarrollada a partir de la evolución del sistema lingüístico en el que fue originalmente creado; hacerlo desde Latinoamérica añade a esa dificultad la necesidad de inscribirlo en coordenadas históricas y culturales diferentes a las peninsulares, pero a la vez configuradas a partir del desarrollo del sistema literario que las versiones españolas precedentes de ese mismo texto contribuyeron a formar ${ }^{3}$. Toda traducción de un poema de Catulo se cimienta sobre determinados presupuestos acerca de su carácter de clásico, de la noción de literatura y de los rasgos propios del género poético que intervienen tanto en la adopción de cierta variedad lingüística, como en la preferencia por el léxico de determinados registros y sociolectos en el texto resultante. En uno de sus ensayos, Borges (1974: 239) afirma que toda modificación del comienzo de El Quijote le parece sacrílega y atribuye esa sensación a su ejercicio congénito del español, precisamente para oponerla a la librería internacional de obras en prosa y verso, que su oportuno desconocimiento del griego le ofrece para aproximarse a la Odisea. No obstante, la diversidad presente en esos numerosos anaqueles de versiones homéricas ha prescindido, históricamente, de muchas variedades, registros y sociolectos. Un poema como la Ilíada, cuya estatura épica, carácter clásico y estilo elevado le han sido inculcados a la tradición occidental desde la misma Poética de Aristóteles, no podría aceptar -acaso ni siquiera como irreverente parodia- una traducción que proponga: «Che, diosa, cantá, la bronca de Aquiles». En tal sentido, el contexto socio-histórico y cultural en que trabaja un traductor argentino, chileno o mexicano resulta significativo, pero no por determinar de manera lineal el desarrollo de su tarea, sino debido al complejo entramado de relaciones que provee como marco para las eventuales decisiones glotopolíticas ${ }^{4}$ adoptadas.

\footnotetext{
${ }^{3}$ Cf., a su vez, lo que sostiene Sverdloff (2013: 163): «Cuando Marrón o Cófreres-Mércuri traducen los epigramas de Marcial, ni ellos ni sus lectores están demasiados seguros de cómo debe sonar una traducción argentina. Este primer encuentro entre Marcial y el horizonte de traducción argentino no tendrá demasiadas posibilidades de ser "etno-céntrico", en la medida en que no hay una tradición fuerte sobre la que tal anexión etnocéntrica podría fundarse: una situación comparable, en algún sentido, a la que describe Berman para la Alemania del siglo XVIII. Asimismo, la presencia en nuestro horizonte de ediciones y traducciones filológicas de diversas lenguas dificulta que las traducciones, por más que operen en ellas las inevitables tendencias deformantes, se conviertan en una mera "anexión", dado que siempre serán leídas contra otras traducciones.»

${ }^{4}$ Adherimos a la reformulación realizada por Arnoux (2000) a partir de la definición clásica de Guespin \& Marcellesi (1986), entendiendo por «acciones glotopolíticas» al conjunto de intervenciones explícitas,
} 
En la introducción a su edición bilingüe de la obra de Catulo, publicada en México en 1969, Bonifaz Nuño afirma haberse atenido lealmente, en cuanto le fue posible, a la más estricta literalidad (1969: 70-71). Si bien se explaya acerca de los aspectos métricos considerados para la elaboración de su versión rítmica, no hace referencia al tipo de estrategias adoptadas para ejercer su lealtad a la mayor literalidad posible, por lo que parece invitarnos a inferirlos a partir de la confrontación de los originales latinos con sus traducciones. Observemos, entonces, a modo de ejemplo, los dos versos iniciales del carmen 37 de Catulo en su versión mexicana:

Salaz tienda y vosotros, contubernales,

de los hermanos con píleo al pilar nono (Bonifaz Nuño, 1969)

Salax taberna uosque contubernales,

a pilleatis nona fratribus pila (Catull. 37, 1-2)

Pese a tamaña demostración de lealtad al original, Bonifaz Nuño parece haber sentido la necesidad de no abandonar totalmente a sus contemporáneos, por lo que anexa a estos versos algunas notas incluidas al final del libro (1969: 159). De esa manera, informa que la «salaz tienda» era «uno de los lugares donde se reunía la juventud galante de Roma». Luego, aclara que «los hermanos con píleo» son «Cástor y Pólux, a quienes se representaba cubiertos de bonetes cónicos de fieltro» y cuyo «templo se alzaba en el lado sur del Foro». Por último, explica que el texto latino hace referencia «a la tienda indicada por el pilar noveno a partir del templo de los Dioscuros», porque frente a cada una «había una columna que servía como anuncio».

En la introducción, Bonifaz Nuño no menciona ninguna de las traducciones castellanas de Catulo que para entonces habían sido ya editadas en España, tales como la de Juan Petit (1950), la de Miguel Dolç (1963) y la de Víctor José Herrero Llorente (1967). En cambio, sí alude a la versión mexicana publicada por Joaquín Demetrio Casásus (1905), quien proponía, para estos mismos dos versos, la siguiente traducción:

comportamientos espontáneos, actividades epilingüísticas y prácticas metalingüísticas que contribuyen a la conformación, reproducción o transformación de las relaciones sociales y de las estructuras de poder, ya fuere a escala local, nacional, regional o mundial. 
Taberna, que al pilar noveno te hallas,

de Cástor y de Pólux junto al templo,

y vosotros, que sois sus parroquianos (Casásus, 1905)

La traducción de Casásus, incluida en una edición también bilingüe, carece de todo tipo de notas al pie y al final, no sólo para este pasaje, sino para la totalidad de los poemas del libro. Emitiendo un juicio de valor apresurado, podría pensarse que la versión de Bonifaz Nuño, cuya obstinada pretensión de lealtad cuasi-etimológica con relación al original más que esclarecer oscurece, no realiza ningún aporte sustancial a la versión propuesta más de sesenta años antes por Casásus. No obstante, observemos lo que sucede con la traducción de los tres versos siguientes:

¿pensáis solos tener el privilegio

de ser hombres y amar a las mujeres?

¿y nomás para eunuco me creéis bueno? (Casásus, 1905)

¿juzgáis que tenéis vergas vosotros solos;

que, con cuanto hay de niñas, sólo os es lícito fornicar,

y juzgar a los otros, chivos? (Bonifaz Nuño, 1969)

solis putatis esse mentulas uobis,

solis licere quicquid est puellarum

confutuere et putare ceteros hircos? (Catull. 37, 3-5)

Confrontadas ambas versiones, la de Bonifaz Nuño no parece ya tan prescindible, puesto que no recurre a eufemismos ni omisiones. Incluso su enfática voluntad de lograr una traducción lo más literal y leal posible al original resulta más comprensible, ya que el método adoptado por Casásus partía de un propósito distinto, caracterizado de la siguiente manera en su introducción:

traducir literalmente todas las expresiones obscenas que el poeta emplea me hubiera exigido adoptar un lenguaje impropio de la poesía moderna e infringir las reglas más elementales del buen gusto. Por fortuna 
encontré una solución que me permitiera no dejar en el olvido algunas de las joyas más exquisitas del primero de los poetas epigramáticos latinos, y que al mismo tiempo me evitara emplear palabras que desdijeran del refinamiento de nuestra cultura social actual. (...) Mi invariable propósito ha sido dejar siempre vivas las ideas, (...) expresándolas con un lenguaje adecuado. (Casásus 1905: 8-9).

El procedimiento descripto explica el carácter eufemístico de su traducción de los tres versos citados, donde se omiten los términos mentulas y confutuere. Bonifaz Nuño, en cambio, traduce el primero por «vergas» y el segundo por «fornicar»: dos términos casi técnicos, sin estricto anclaje local y rigurosamente consignados por el Diccionario de la Real Academia Española (DRAE). Nótese, no obstante, que las tres traducciones peninsulares precedentes, publicadas bajo la dictadura franquista, eran mucho más conservadoras en la traducción de este pasaje:

¿os figuráis que sólo vosotros sois hombres, y que sólo a vosotros está permitido hacerse con cuantas mozas hay... (Petit, 1950)

¿os figuráis que sólo vosotros sois realmente hombres, que sólo vosotros tenéis el derecho de haceros vuestras todas las mozas... (Dolç, 1963)

¿creéis que sois los únicos que tenéis miembros viriles, los únicos con derecho a desflorar a cuantas doncellas existen... (Herrero Llorente, 1967)

Los lectores españoles tuvieron que esperar hasta la aparición de la versión de Ramírez de Verger -que data del año 1988 y fue la primera posterior a la caída del régimen de Franco- para ver materializado el abandono de los circunloquios emprendido casi veinte años antes por Bonifaz Nuño en tierras mexicanas:

¿creéis que sólo vosotros tenéis verga

y que sólo a vosotros os está permitido joder

a todas las jóvenes (Ramírez de Verger, 1988)

En 1905, Casásus había prescindido en su versión incluso del adjetivo salax que acompaña al sustantivo taberna en el texto original. Bonifaz Nuño, en cambio, traduce 
«tienda» por taberna, no sólo en este pasaje, donde recurre al adjetivo «salaz» para adjetivarla, sino todas las veces que aparece, de manera sistemática. Las tabernae romanas no eran necesariamente «tabernas», sino más bien un tipo de local similar a los actuales comercios minoristas, cuyos rubros abarcaban desde alimentos a diversos servicios. Una clase especial dentro del grupo eran las tabernae uinariae, que se especializaban en el consumo de vino y comida. A esta clase de sitio alude Catulo en su poema, adjetivándolo como salax, es decir, propenso o predispuesto a la lujuria. Casásus directamente omite este adjetivo, acaso asumiendo que toda «taberna» -en tanto sitio asimilado en México con el cabaret francés- era de por sí un lugar proclive a excesos de esa naturaleza. Las tres traducc«iones peninsulares ya publicadas cuando Bonifaz Nuño edita la suya, en cambio, optan por el término «taberna», adjetivado como «indecente» (Petit, Dolç) o «lúbrica» (Herrero Llorente). A su vez, mientras que los literales «contubernales» de la edición de Bonifaz Nuño seguían siendo, en la de Petit, los mismos «parroquianos» de la traducción de Casásus; en la de Dolç habían devenido ya simplemente «los que en ella os reunís» y habían pasado a ser incluso «contertulios» en la de Herrero Llorente. Casásus traduce «taberna», sin necesidad de adjetivo alguno. Bonifaz Nuño opta por «tienda» y la adjetiva como «salaz», pero aclara en sus notas que se trataba «de uno de los lugares donde se reunía la juventud galante de Roma», elevando falsamente el estatus social de ese sitio, al que las versiones peninsulares del siglo XXI llegan incluso a traducir, con mayor precisión, directamente como tugurio »(Alonso Gamo, 2004«) o «garito» (González Iglesias, 2006). No obstante, en un contexto donde Bonifaz Nuño no vacila en utilizar términos etimológicamente equivalentes a los latinos -como «contubernales», «salaz», «píleo»y «pilar nono»- la cabal precisión de la palabra «tienda», en detrimento de la homógrafa «taberna», resulta una decisión particularmente interesante, sobre todo cuando el único traductor mexicano anterior, Casásus, adopta sin vacilar el otro vocablo. Todo indica que Bonifaz Nuño no consideraba posible realizar una traducción lealmente literal en aquellos casos donde el término español más parecido al original no reflejaba cabalmente el mismo significado que aquél en su contexto histórico.

Por razones de extensión, sería imposible seguir analizando de manera tan minuciosa su traducción completa del poema. No obstante, nos interesa señalar la presencia de un término en particular, que parece ser el único estrictamente local, o al menos 
semánticamente distante del sentido que le asigna el $D R A E$. Se trata del diminutivo «chiquitos», con el que Bonifaz Nuño traduce la palabra latina pusilli. Casásus había optado por «vulgares», Petit por «cualesquiera», Dolç por «pelagatos» y Herrero Llorente por «mezquinos». Otras traducciones peninsulares posteriores eligen «apocados»(Torres Bejar, 1969), «enanos» (Álvarez Ezquerra, 1993), «donnadie» (Soler Ruiz, 1993), «miserables» (González Galicia, 2002), «larvas viles» (Alonso Gamo, 2004), «tan mierdas» (González Iglesias, 2006) o «insignificantes» (Pérez Vega, 2008). Bonifaz Nuño, acaso priorizando la dimensión más concreta del insulto, recurre a la palabra «chiquitos» para referirse a los rivales de Catulo. En latín, pusilli es diminutivo de pullus, término que alude a un brote vegetal o a la cría animal de cualquier especie, y se utiliza también, por extensión, para hacer referencia a los niños o a los jóvenes. «Chiquitos» responde perfectamente a ese valor, en especial en América, donde la distribución del sufijo «-ito» siempre estuvo más expandida que en España. No se trata del único caso del libro en que Bonifaz Nuño vierte al español un diminutivo recurriendo a ese infijo. No obstante, elige traducir la única otra ocurrencia de pusillum, en el sintagma caput pusillum, como «cabeza muy chiquitilla», y no «chiquita», ni «chiquitita», ni «muy chiquitita». Por otra parte, en algunas ocasiones alterna el uso de uno y otro sufijo formativo para los mismos términos. Por ejemplo, al traducir labelli dos veces como «labiecitos»y otras dos como «labiecillos»; o bien, al traducir lecticulus como «lechillo» en un caso y lectulus como «lechito» en otros dos. Todo parece indicar, entonces, que tras la traducción de pusilli como «chiquitos» (en el sentido de «son chiquitos, insignificantes, no existen») emerge un uso regionalmente anclado y distante de la variedad peninsular, mayormente respetada en el resto de las decisiones adoptadas en el libro, cuya expresión equivalente sería, para este caso, «sois pequeños».

La edición bilingüe de los poemas de Catulo a cargo de Bonifaz Nuño forma parte de la Bibliotheca Scriptorum Graecorum et Romanorum Mexicana, proyecto editorial surgido en el año 1944,

con el propósito de poner a la mano de los estudiosos de la filosofía, la ciencia y la literatura grecolatinas textos clásicos en la lengua original en la que fueron escritos, acompañados de traducciones y estudios realizados por reconocidos investigadores de la UNAM (Pereira, 2004: 139).

El carácter bilingüe de la colección responde también a un segundo objetivo, quizás 
menos claro en sus inicios, pero explícito en varias de sus obras, consistente en ayudar a alumnos de griego y de latín a aprender esas lenguas mediante la confrontación de los textos con sus traducciones (Villaseñor Cuspinera, 2005: 263). Si bien, a diferencia de México, Argentina no ha contado con un proyecto editorial universitario orientado a la traducción y difusión sistemática de los clásicos grecolatinos, a principios del siglo XXI las editoriales Losada y Colihue comenzaron a fomentar la publicación de nuevas traducciones de textos griegos y latinos realizadas por especialistas argentinos. Recién en el año 2008, precisamente en el marco de la colección Colihue Clásica, se publica la primera traducción integral de la obra de Catulo realizada en nuestro país ${ }^{5}$, como edición bilingüe, a cargo de Lía Galán, investigadora del CONICET y docente de la Universidad Nacional de La Plata. Observemos, entonces, los primeros cinco versos del poema que ya hemos analizado, pero ahora en la versión propuesta por Galán:

Indecente taberna y vosotros, sus compañeros,

que estáis junto a la novena columna después de los hermanos del gorro frigio,

¿pensáis que vosotros solos tenéis vergas,

que para vosotros solos es lícito a cuantas muchachas haya

coger y considerar a los demás cabrones? (Galán, 2008)

Podríamos pensar que la acumulación léxica de términos como «taberna», «vergas»y «cabrones», sumada a la adopción del pronombre de segunda persona plural «vosotros» para la conjugación verbal, inscriben este texto en una suerte de constructo lingüístico panhispánico ${ }^{6}$. No obstante la adopción del verbo «coger» para traducir confutuere desarticula toda pretensión de neutralidad.

A diferencia de la versión de Bonifaz Nuño, que contaba con un antecedente mexicano y otros tres peninsulares (todos ellos en mayor o menor medida eufemísticos), la versión de Galán se encuentra precedida por al menos otras nueve traducciones más, editadas en España, en las que el carácter «técnico» de términos como «verga»y «fornicar»,

\footnotetext{
${ }^{5}$ La segunda, a cargo de Marta Elena Caballero (2010) se enmarca en un proyecto editorial de la Universidad Católica de Córdoba, pero excluye los carmina 61-68.

${ }^{6} \mathrm{Si}$ bien no nos ocupamos aquí del análisis del uso pronominal de la segunda persona singular en las traducciones de los textos clásicos, remitimos a Marrón (2014: 150), donde puede leerse una breve reflexión sobre el tema.
} 
propuestos en 1969 por el mexicano, han ido tornándose cada vez más localmente anclados: Torres Bejar (1969) los traduce como «miembro viril» y «violar»; Ramírez Verger (1988), como «verga» y «joder»; Rodríguez Tobal (1991), como «polla»y «tirarse»; Álvar Ezquerra (1993), como «méntulas» y «joder»; Soler Ruiz (1993), como «cojones» y «joder»; González Galicia (2002), como «polla» y «joder»; Alonso Gamo (2004), como «membrudos» y «poseer»; González Iglesias (2006) y Pérez Vega (2008), como «pollas»y «follar ». Ninguna objeción cabría entonces a la adopción del verbo «coger» para traducir confutuere, excepto porque, articulado con el pronombre de segunda persona plural «vosotros», da lugar a una construcción híbrida e inverosímil, que -como el sintagma «sois chiquitos»- no podría concebirse fuera del texto propuesto. El DRAE registra como acepción número trigésimo primera del verbo «coger» la de «realizar al acto sexual», pero aclara que se trata de un vulgarismo utilizado en América Central, Argentina, Bolivia, México, Paraguay, República Dominicana, Uruguay y Venezuela. La intencionalidad subyacente tras la adopción de ese verbo en la traducción de Galán se explica, no obstante, a partir de lo señalado por ella misma en la introducción del libro, cuando afirma:

La palabra o expresión obscena es local y temporaria, y esto vale para la lengua que se traduce y para la lengua en la que se traduce. (...) Varían sin duda del latín al castellano, pero también varían según se trate del castellano peninsular, rioplatense o centroamericano. En tal sentido, creemos inevitable aunque no completamente feliz, recurrir a una especie de koiné o lenguaje común de vulgarismos, comprensible en todas las regiones del español, aunque no sea esta la única posibilidad que a veces se presenta al traductor. Si por scortillum traducimos «putita» (que creemos un poco mejor que «golfilla» y muy superior a «pellejuela») esto no quiere decir que hayamos encontrado la equivalencia precisa -suponemos que es muy difícil que alguien hable estrictamente así en América, ya que probablemente usaría un vulgarismo local más elocuente- sino una forma aceptable de solucionar el caso. (Galán, 2008: LVII)

Ninguno de los libros en que aparecieron las tres traducciones latinoamericanas del carmen 37 de Catulo a las que nos hemos referido pretendieron ser-como se desprende de lo señalado por Casásus, Bonifaz Nuño y Galán en sus respectivas introduccionesversiones «mexicanas»o «argentinas» de la obra de Catulo, sino más bien inscribirse en una lengua que pudiera entenderse tanto en España como en cualquier país latinoamericano. En tal sentido, la adopción del pronombre de segunda persona plural «vosotros», en detrimento de «ustedes», resulta casi sintomática del presupuesto 
panhispánico subyacente en todos los casos. En primer lugar, porque ninguno de los tres traductores consideró necesario brindar explicaciones respecto a por qué decidió adoptarla. Y, en segundo término, porque a diferencia de lo que sucede en la comunidad lingüística castellana del territorio español, donde ambos usos poseen una distribución diferenciada, en términos de mayor o menor gradación de formalidad, en Latinoamérica resulta privativo el uso de «ustedes» en todos los casos.

Si los términos «coger» $\mathrm{y}$ «chiquitos» se usan prácticamente en todas las comunidades de habla españolas no peninsulares $\mathrm{y}$, sin embargo, emergen como rarezas en las traducciones de Galán y Bonifaz Nuño respectivamente, ¿por qué la insistencia en prescindir del uso del pronombre de segunda persona plural «ustedes», que resulta comprensible en toda Latinoamérica y también en España? Dos traducciones latinoamericanas relativamente recientes -una publicada en Chile, en 2010, y la otra en Argentina, en 2012- parecen haber sido las primeras en abandonar el «vosotros»y preferir el «ustedes». En la versión chilena de Leonardo Sanhueza, la traducción de los primeros cinco versos del carmen 37 de Catulo es la siguiente:

Parroquianos de ese tugurio decadente

que está a nueve puertas de los Hermanos con Gorra,

¿qué se creen: que ustedes nomás tienen verga

y que pueden tirarse a todas las lolitas... (Sanhueza, 2010)

El mismo fragmento de ese poema, en la versión argentina de mi autoría, aparece traducido del siguiente modo:

Le hablo a ese bar promiscuo y a ustedes, que se juntan ahí, unas nueve columnas más allá del templo de Cástor y Pólux: ¿se creen los únicos que tienen pija?, ¿piensan que ustedes solos pueden cogerse a todas las minas... (Marrón, 2012)

El fenómeno observable en estas últimas dos versiones coincide con el presente en la publicada en 2006, por González Iglesias en España, donde el uso del léxico propio de la variedad peninsular también impide toda posible inscripción del texto resultante en el constructo lingüístico -pretenciosamente- panhispánico denominado «español neutro». Así como Sanhueza habla de «lolitas» para traducir puellae y en mi traducción yo 
recurro al término «minas», González Iglesias las llama «tías». Mentula, el mismo término que yo traduzco «pija» y Sanhueza «verga», es traducido «polla» por González Iglesias. De igual modo, la taberna, que resulta «bar» y «boliche» en mi versión, es «tugurio» $\mathrm{y}$ «cantina» en la chilena, pero también «garito» en la española.

Como ya hemos señalado, traducir a Catulo desde Latinoamérica y en el siglo XXI plantea un doble desafío: el de trasladarlo a una lengua desarrollada a partir de la evolución de aquella en la que fue creado, pero también el de inscribirlo en el horizonte de sistemas literarios que tienen ya una identidad propia, y en los que las obras de autores internacionalmente reconocidos -como Juan Rulfo, Pablo Neruda o Julio Cortázar, por mencionar sólo tres- prestigiaron desde hace años el uso del pronombre de segunda persona plural «ustedes», cristalizado mucho tiempo antes también en la lengua oral (Moreno de Alba, 2010: 462). ¿Por qué, entonces, recurrir a una conjugación verbal de segunda persona plural, que no sólo resulta ajena en nuestras tierras desde hace años, sino que, a su vez, colisiona con toda posibilidad de anclar lexicalmente nuestras versiones, como sí lo hacen, en cambio, los más recientes traductores peninsulares?

\section{Addenda}

La selección de textos que conforma Habeas Corpus. Latín, sexo y traducción no surgió con el propósito de ser publicada bajo la forma de un libro, ni mucho menos con el ámbito de la enseñanza universitaria como horizonte, sino más bien de manera azarosa, a partir de una informal conversación de sobremesa. El primer sábado de julio del año 2010, una colega -que había sido también mi profesora durante la carrera de gradoaludió al particular contenido de un epigrama de Marcial desconocido por mí -y cuya referencia exacta ella no recordaba- para contar que había sido incluido dentro del corpus de textos trabajados en las clases de latín cuando ella era estudiante. Ante mi incredulidad $-\mathrm{y}$ la del resto de los comensales- tanto con respecto al tópico supuestamente abordado en el poema, como a la posibilidad de que la traducción de un epigrama de esas características hubiera sido leído en voz alta en una clase de latín de la Universidad Nacional del Sur, revisamos los dos tomos de Marcial que estaban en mi 
biblioteca. Fue en vano, esa noche no pudimos encontrarlo, pero al día siguiente, ya a solas y gracias a la base de datos de textos latinos del Packhardt Humanities Institute, logré determinar que se trataba del epigrama dieciocho del séptimo libro. Tras una primera lectura del texto, busqué la versión castellana, en el segundo tomo de la edición de Antonio Ramírez de Verger para Gredos, que aparece allí enigmáticamente titulada «Contra Gala, hermosa pero tonta». Gala, la joven construida como destinataria en ese satírico epigrama, es, en efecto, muy bella, pero no tonta; o, en todo caso, ese aspecto no motiva las críticas dirigidas a ella por la voz poética, como puede observarse en la traducción:

Si tienes una cara, de la que ni una mujer podría hablar,

si ninguna mancha hay en tu cuerpo.

¿por qué te extrañas de que tan pocos folladores te deseen

y vuelvan otra vez? Tu defecto. Gala, no es pequeño.

Cuantas veces me inicié en la faena y nos meneamos con las

ingles pegadas, el coño no calla, tú eres la que callas.

Los dioses hicieran que hablaras tú y callara aquél:

me fastidia la garrulidad de tu coño.

Preferiría que te peyeras: que esto, dice Símaco, no perjudica

y es cosa esa que mueve a risa a la vez.

¿Quién puede reírse de los ruidos de un coño loco?

Cuando suena éste, ¿qué polla y cabeza no se vienen abajo?

Di al menos algo y mete ruido al son de tu coño gritón,

y si eres tan muda, aprende a hablar aunque sea por allí. (Ramírez de Verger, 2001, T. II:15)

Marcial satiriza la garrulitas cunni de Gala, y la versión de Ramírez de Verger reproduce sin eufemismos la idiosincracia del epigrama. Sin embargo, el léxico adoptado en su traducción para hacer referencia a los aspectos sexuales y escatológicos me resultaba distante. Tanto el subjuntivo «peyeras» (pēeère), como los sustantivos «folladores»(fututori), «coño» (cunnus) y «polla» (mentula) -propios de un registro informal tanto en su lengua de origen, como en la del traductor- remiten a un horizonte 
lingüístico demasiado peninsular para el hablante argentino, cuya lengua coloquial supone mayor proximidad con equivalentes terminológicos tales como «pedorrearas», «cogedores», «concha»y «pija», respectivamente; con la salvedad de que el segundo reviste más bien una función adjetiva en nuestro léxico, que privilegia la variante analítica en detrimento de la sintética -«los que (te) cogen»- para los contextos que expresan agentividad. A partir de la percepción de que esos cuatro términos -explícitos, pero propios de otra variedad lingüística- constituían las instancias más distantes de la versión peninsular, me propuse intentar lo que terminaría siendo un intuitivo primer ejercicio de traducción, orientado a reducir la brecha lingüística entre el texto latino y sus potenciales lectores.

Las redes sociales, que contribuyeron a la circulación de mi versión de ese epigrama de Marcial $^{7}$, también dieron lugar a que distintas personas comenzaran a solicitarme nuevas traducciones de otros textos, cuya temática o crudeza descriptiva se hallaba en consonancia con el que yo había compartido originalmente; y así, poco a poco, se fue configurando un corpus «a demanda», en el que algunos poemas de Catulo, Horacio, Claudiano y Ausonio se sumaron a otros numerosos de Marcial. Pocos meses después, tanto los organizadores de la Feria de Editoriales Autogestionadas de la ciudad de Bahía Blanca, como los de la Feria del libro de Sierra de la Ventana me invitaron a leer públicamente esas traducciones, entre las que ya era incluso posible operar una selección, debido a su sostenido crecimiento. Ante la incomodidad que me generaba leer en público textos de ese tenor, recurrí al auxilio de la actriz Elena Bonora, que aceptó recitarlos conmigo, mediante un contrapunto en el que yo, vestida con un atuendo formal, leía las versiones latinas, y ella, caracterizada de romana, los textos traducidos. El resultado de la primera representación fue catastrófico: muchos espectadores pensaron que lo que yo leía no era latín y lo que ella recitaba no tenía la más mínima relación con lo leído antes por mí; no obstante ello, reían, aplaudían estrepitosamente y preguntaban dónde podían adquirirlos impresos. La segunda vez, pese a incorporar una breve introducción que pretendió dejar en claro la naturaleza del trabajo previo y el planteo de la representación, tampoco nos fue mucho mejor. Como corolario de la catástrofe, tres de los editores presentes, que habían escuchado las

\footnotetext{
${ }^{7}$ Cf., por ejemplo, la sección "Ecos" del blog Guarradas Puéticas de la Antigüedá Grecolatina, http://www.guarradaspueticas.blogspot.com.ar.
} 
lecturas, se mostraron muy interesados en publicar las traducciones; y el del sello editorial Vox Senda -que había editado ya en 1999 el Catulito del poeta Sergio Raimondi ${ }^{8}$ - logró convencerme.

A partir de su publicación, en el año 2012, los textos que integran Habeas Corpus. Latín, sexo y traducción hicieron un recorrido propio ${ }^{9}$. No sé si actualmente los traduciría del mismo modo, es decir, despojados prosaicamente de toda poesía; y tampoco sé si volvería a publicarlos sin un prólogo ampliado que abarcara también otras complejidades de índole no necesariamente léxica y pronominal, sino ideológica y política. En el Epodo 8 de Horacio incluido en el libro, por ejemplo, se presenta a una mujer que excede el límite generacional impuesto por la sociedad a su apetito sexual. Todo monstruo advierte, es símbolo de los límites que no se deben cruzar; en este caso, el monstruo es ella. El poeta la describe, la denigra, la torna risible; porque la risa es sanción y exorciza el miedo de caer uno mismo en el ridículo. Durante siglos, los traductores resolvieron la existencia de ese poema mediante el ejercicio de una suerte de desplazamiento o sinécdoque: transformaron el texto mismo en monstruo. Lo dejaron en latín, al lado de una hoja en blanco, sin traducción, como advertencia de lo que no puede decirse, de lo que no debe ponerse en palabras, de lo que es imposible traducir a la lengua propia porque sólo puede expresarse en otra ${ }^{10}$. No conformes con ello, lo llenaron de comentarios lapidarios: «este dístico, el más puerco que se hizo jamás», dice Javier de Burgos (1821: 350) en una de las notas de su edición castellana; nota que acompaña el texto latino que no se traduce, porque «el temor de mancharse con tales porquerías impide ocuparse de ninguna interpretación»(de Burgos, 1821: 351). A la distancia, uno lee ese epodo y encuentra un vientre fofo (uenter mollis), un culo flaco (aridas natis uelut crudae bouis), unas tetas largas (mammae putres equina quales ubera), arrugas (rugis), un diente cariado (dens ater), piernas flacas (femur additum), tobillos anchos (tumentibus suris), y un hombre -un yo poético- al que no excita ese cuerpo y demanda sexo oral para alcanzar la erección (ore adlaborandum est tibi). A la

\footnotetext{
${ }^{8}$ Cf., a su vez, Raimondi (2017), la nueva edición ampliada del mismo libro.

${ }^{9}$ Cf., entre otras repercusiones Erbetta (2012), Schinca (2013), y la sección "Ecos" del blog Guarradas Puéticas de la Antigüedá Grecolatina, http://www.guarradaspueticas.blogspot.com.ar.

${ }^{10}$ Cf., por ejemplo, Roberts (2008, 294-295): «Translators of [greek and latin classical] texts regarded as obscene regularly cite the approach of the French as a kind of antitype to English reticence -for better or for worse. (...) It is clear that from the English-speaker's point of view French is envisioned as the all-too appropriate target language where obscenity is concerned; too appropriate, indeed, for decent use.»
} 
distancia, reitero, uno lo lee y piensa: qué chiquito el monstruo, qué diminuto el monstruoso texto al que tanto miedo le tuvieron ciertos traductores. Las mujeres de las que hablaba Horacio (y muchos otros sujetos cuyas prácticas son satíricamente sancionadas en los textos que integran Habeas Corpus), en cambio, siguen siendo ridiculizadas y demonizadas por otros discursos sociales, menos poéticos, ya no en latín, pero no por eso menos agresivos y violentos. A veces me pregunto si publicar Habeas Corpus no ha sido una forma (también) de contribuir a perpetuar la sanción y la risa expiatoria. Cada tanto esa culpa y yo fumamos un rato en el patio; y después me olvido por varios meses de la cuestión.

\section{Bibliografía}

Arnoux, E. (2000). "La Glotopolítica: transformaciones de un campo disciplinario" en Lenguajes: teorías y prácticas. Buenos Aires: Gobierno de la Ciudad de Buenos Aires, 95-109.

Borges, J. L. (1974). “Las versiones homéricas” en Discusión. Buenos Aires: Emecé, O.C. T. I: 239- 243.

Erbetta, E. (2013). Una historia de la guarrada. Diario Página/12. Suplemento Cultura y Espectáculos. Buenos Aires, 8 de junio, año 27, n 8847, Buenos Aires, 13.

Guespin, L. y Marcellesi, J.-B. (1986). Pour la Glottopolitique. En Revue Langages ( ${ }^{\circ}$ 83), 5-34. Paris: Armand-Colin.

Marrón, G. A. (2014). El rapto de Helena de Blosio Emilio Draconcio. En Circe de Clásicos y Modernos ( $\left.{ }^{\circ} 18\right)$, 147-169. La Pampa: Universidad Nacional de la Pampa.

Moreno de Alba, J. G. (2010). Notas sobre la cronología de la eliminación de vosotros en América. En Castañer Martín, R. M. y Lagüén Gracia, V. (Coord.) De moneda nunca usada: Estudios dedicados a José Ma Enguita Utrilla. Zaragoza: Instituto Fernando el Católico, 461-470.

Pereira, A. (2004). Diccionario de Literatura Mexicana. Siglo XX. México: UNAM.

Roberts, D. (2008). Translation and the 'Surreptitious Classic': Obscenity and 
Translatability. En Lianeri, A. \& Zajko, V. (Ed.) Translation \& the Classic. Identity as Change in the History of Culture. Oxford: Oxford University Press, 278-311.

Schinca, G. (2013). Alta cochinada. Latín, sexo y traducción (y sexo). En El toldo de Astier. Propuestas y estudios sobre enseñanza de la lengua y la literatura ( $\left.{ }^{\circ} 7\right), 129$ 133. La Plata: Universidad Nacional de La Plata.

Sverdloff, M. (2013). Reflexiones sobre Antoine Berman y los epigramas: Marco Valerio Marcial en la lengua literaria argentina del siglo XXI". En Revista Letral (n ${ }^{\circ}$ 11), 156-175. Granada: Universidad de Granada.

Villaseñor Cuspinera, P. et al. (2005). Presentación de la Bibliotheca Scriptorvm Graecorvm et Romanorvm Mexicana. En Nova Tellus ( $\mathrm{n}^{\circ}$ 23), 251-268. México: UNAM.

\section{Traducciones referidas}

Alonso Gamo, J. M. (2004). Catulo. Poesías completas. Guadalajara: AACHE.

Alvar Ezquerra, A. (1993). Poesía de amor en Roma. Catulo. Tibulo. Lígdamo. Sulpicia. Propercio. Madrid: Akal.

Bonifaz Nuño, R. (1969). Catulo. Carmenes. México: UNAM.

Caballero, M. E. (2010). Catulo. Carmina Minora. Córdoba: EDUCC.

Casásus, J. D. (1905). Las Poesías de Cayo Valerio Catulo. México: Ignacio Escalante.

De Burgos, J. (1821). Las poesías de Horacio. Traducidas en versos castellanos, con notas y observaciones críticas. Madrid: Imprenta del Collado.

Dolç, M. (1963). Catulo. Poesías. Barcelona: Alma Mater.

Galán, L. M. (2008). Catulo. Poesía Completa. Buenos Aires: Colihue.

González Galicia, R. (2002). Catulo. Catulli Carmina. Madrid: Babab.

González Iglesias, J. A. (2006). Catulo. Poesías. Madrid: Cátedra.

Herrero Llorente, V. J. (1967). Catulo. Poesías. Madrid: Aguilar. 
Marrón, G. A. (2012). Habeas Corpus. Latín, sexo y traducción. Bahía Blanca: Vox Senda.

Pérez Vega, A. (2008). Poemas de Gayo Valerio Catulo. Sevilla: Orbis Dictus.

Raimondi, S. (1999). Catulito. 14 Endecasílabos y el Talo Maricón de Catulo, Bahía Blanca, Vox Senda.

Raimondi, S. (2017). Catulito. 23 Endecasílabos y el Talo Maricón de Catulo, Bahía Blanca / Santa Fe: Vox Senda / Neutrinos.

Ramírez de Verger, A. (1988). Catulo. Poesías, Madrid: Alianza.

Ramírez de Verger, A. (2001). Marcial. Epigramas, Madrid: Gredos.

Rodríguez Tobal, J. M. (1991). Catulo. Poesía Completa. Madrid: Hiperión.

Sanhueza, L. (2010). Leseras. Santiago de Chile: Ediciones Tácitas.

Soler Ruiz, A. (1993). Catulo: Poemas. Tibulo: Poemas. Madrid: Gredos.

Torrens Béjar, J. (1969). Obras Poéticas. Catulo y Tibulo. Barcelona: Iberia. 